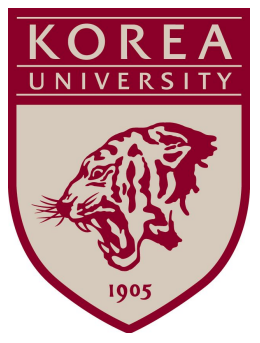

Discussion Paper Series

No. 1010

May 2010

\title{
Licensing a common value innovation when signaling strength may backfire
}

\author{
Cuihong Fan, Byoung Heon Jun \\ and Elmar G. Wolfstetter
}

The Institute of Economic Research - Korea University

Anam-dong, Sungbuk-ku, Seoul, 136-701, South Korea, Tel: (82-2) 3290-1632, Fax: (82-2) 928-4948 


\title{
Licensing a common value innovation when signaling strength may backfire*
}

\author{
Cuihong Fan \\ Shanghai University of Finance and Economics \\ Byoung Heon Jun \\ School of Economics ${ }^{\dagger}$ \\ Korea University, Seoul \\ Department of Economics
}

\author{
Elmar G. Wolfstetter \\ Humboldt-University at Berlin and \\ Korea University, Seoul ${ }^{\S}$
}

May 4, 2010

\begin{abstract}
This paper reconsiders the licensing of a common value innovation to a downstream duopoly, assuming a dual licensing scheme that combines a first-price license auction with royalty contracts for losers. Prior to bidding firms observe imperfect signals of the expected cost reduction; after the auction the winning bid is made public. Bidders may signal strength to their rivals through aggressive bidding, which may however backfire and mislead the innovator to set an excessively high royalty rate. We provide sufficient conditions for existence of monotone bidding strategies and for the profitability of combining auctions and royalty contracts for losers.
\end{abstract}

KEYWORDS: Patents, licensing, auctions, royalty, innovation, R\&D, mechanism design.

JEL ClassifiCATIONS: D21, D43, D44, D45.

\section{Introduction}

This paper revisits the analysis of the licensing of a non-drastic process innovation by an outside innovator to a Cournot oligopoly. Like in the classical literature on

*We would like to thank Jinwoo Kim, Jae Nahm, and Kiho Yoon for comments. Research support by Korea University and the Deutsche Forschungsgemeinschaft (DFG), SFB Transregio 15, "Governance and Efficiency of Economic Systems," the Humanities and Social Sciences Research Foundation of Chinese Ministry of Education (Grant No. 09YJA790133), and the Leading Academic Discipline Program, 211 Project for SUFE (3rd phase) is gratefully acknowledged.

†Shanghai University of Finance and Economics (SUFE), School of Economics, Guoding Road 777, 200433 Shanghai, China, Email: cuihongf@mail.shufe.edu.cn

¥Department of Economics, Korea University, Sung-buk Ku An-am Dong 5-1, Seoul 136-701, Korea, Email: bhjun@korea.ac.kr

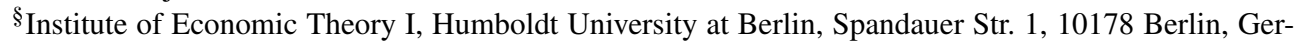
many, Email: wolfstetter@googlemail.com 
patent licensing the cost reduction induced by the innovation is of the common value type. However unlike the classical literature, we assume that this cost reduction is only imperfectly known at the time of bidding for a license, and that observed bids reveal information about firms' private information before the downstream market game is played.

The other novel feature is that we consider a generalized licensing mechanism that combines a first-price license auction with a mandatory royalty contract for those who lose the auction. That royalty contract requires losers to pay a royalty rate per output unit equal to the expected cost reduction inferred from observed bids. The advantage of this scheme is that it allows the innovator to tap into two sources of revenue: the revenue earned in the auction, which is equal to the highest bid paid by the winner, and the royalty income paid by those who lose the auction.

Under complete information, this royalty scheme is evidently more profitable for the innovator than a standard license auction, because there losers' unit cost is the same with and without royalty contract, and therefore equilibrium bidding is unaffected by the royalty contract scheme. However, under incomplete information, the impact of the royalty scheme is more complex. Rational bidders take into account that their bids affect others' beliefs. And their strategic signaling exerts a downward pressure on equilibrium bids, which reduces the expected auction revenue. The question is then whether this reduction in auction revenue either exceeds or falls short of the additional royalty income.

Specifically, adding the royalty scheme gives rise to a dual signaling problem: If a bidder wins the auction, his bid signals his private information both to rival firms and to the innovator, whereas if he loses the auction, his bid exclusively signals his private information to the innovator who sets the royalty rate equal to the expected cost reduction. Bidders take this informational externality into account. In particular, bidders understands that they may gain a strategic advantage in the oligopoly game by an "inflated bid" that signals a higher than true cost reduction, provided their bid happens to win the auction. However, they must also realize that signaling strength may backfire when they lose the auction, and mislead the innovator to set a royalty rate that exceeds the true cost reduction and perhaps also mislead the winner to believe that his expected cost reduction is higher than it is.

Of course, no such "misleading" signaling occurs on the equilibrium path of a separating equilibrium, although the potential to mislead affects the equilibrium bid function. If a separating equilibrium exists, the marginal benefit of signaling a cost reduction that deviates from the true cost reduction must be matched by a corresponding marginal cost in such a way that both kinds of signaling are deterred in all states of the world.

There is large literature on patent licensing in oligopoly by an outside innovator, among which the following contributions are closely related to the present paper.

In their seminal contributions, Kamien (1992), Kamien and Tauman (1984, 1986), Katz and Shapiro $(1985,1986)$ show that auctioning a restricted number of licenses is strictly more profitable for the innovator than other mechanisms, such as pure royalty contracts, fixed-fee licensing, and two-part tariffs. ${ }^{1}$ The limitation of the classical liter-

\footnotetext{
${ }^{1}$ Recently, Sen (2005) shows that if one takes into account that the number of licenses must be an integer,
} 
ature is that it assumes complete information both in the auction and in the subsequent oligopoly game.

The later literature introduced incomplete information but replaced the common value assumption by the private values paradigm. Jehiel and Moldovanu (2000) introduce incomplete information at the bidding stage, combined with complete information in the oligopoly game. They show that patent licensing under incomplete information is a prime example of an auction with negative externalities, where bidders' payoffs depend not only on their type, but also on the type of the player who wins the auction. They claim that in the presence of these externalities the reserve price plays only a limited role because "when the seller sells more often, the buyers are more afraid that the good will fall into the hands of the competitor, and they bid more aggressively" Jehiel and Moldovanu (2000, p. 777).

More recently, Das Varma (2003) and Goeree (2003) reconsider that model under the more plausible assumption that firms do not know each other's private value cost reductions after the auction and before the oligopoly game is played. This introduces the possibility to signal the own cost reduction to rival firms which may interfere with existence of a monotone bidding equilibrium.

Das Varma (2003) considers both Cournot and Bertrand competition (with product differentiation) assuming a linear model. He shows that a separating equilibrium exists under Cournot competition but generally fails to exist under Bertrand competition when goods are substitutes. Whereas Goeree (2003) assumes reduced form payoff functions of the oligopoly "subgames" that are generally satisfied for Cournot but not for Bertrand market games with substitutes, and focuses on a comparison between the three standard auction formats, first-price, second-price, and English auctions.

In a preceding paper, Giebe and Wolfstetter (2008) introduce an optional royalty scheme to the license auction under complete information, and show that such a mechanism is strictly more profitable than the standard license auction, even if the number of auctioned licenses is a choice variable. And in a companion paper Fan et al. (2009a) consider the licensing problem with royalty scheme when cost reductions are specific to the firm who adopts the innovation, in line with the private values case considered by Das Varma (2003), Goeree (2003), Jehiel and Moldovanu (2000).

The main differences between the common value case under incomplete information considered in the present paper, the common value case under complete information, and the private values case can be summarized as follows.

Compared to the common value case under complete information, adding the royalty scheme gives rise to a complex signaling problem because bidders must take into account how their bids affect the beliefs of the innovator, who uses the information revealed by bids to set the royalty rate equal to the inferred, expected cost reduction, and of rival firms who use the information revealed by rival bids to assess their own expected cost reduction. In the face of this signaling issue, one must carefully check whether a separating equilibrium exists at all, and, in assessing the profitability of the

pure royalty contracts can be superior to license auctions. However, this result is again reversed if one generalizes the format of license auctions (see Giebe and Wolfstetter, 2008). See also Sen and Tauman (2007) who analyze an auction of royalty contracts. 
royalty scheme, one must study the trade-off between auction revenue and royalty income that is generally associated with the royalty scheme because the possibility to signal one's private information typically creates a downward pressure on equilibrium bids.

Compared to the private values case, the benefit of adding the royalty scheme is stronger. In particular, in the private values case, existence of a separating equilibrium already fails unless the innovator sets a sufficiently high reserve price, which is typically not an issue in the common values case. To understand the intuitive reason for this fundamental role of the reserve price in the private values case is as follows: a bidder with a low cost reduction may benefit from "low balling" by bidding as if his cost reduction were equal to the lowest value. Because he can thus obtain the right to use the innovation while paying a minimal zero royalty rate. This incentive can only be removed by adopting a high reserve price that makes "low balling" costly. By contrast, in the common value case, the own signal, and in particular the lower signal, has only a relatively small effect on the expected cost reduction and the corresponding royalty rate.

Since the reserve price plays such a fundamental role in the private values case, the comparison of the innovator's expected profits with and without royalty scheme becomes complicated because one must compare profits at the respective optimal reserve prices for which generally no closed form solution can be found.

Another difference is that in the private values case, adding the royalty scheme always reduces the auction revenue, whereas in the common value case that effect is generally smaller and may even be absent altogether. The latter occurs when the highest signal is a sufficient statistic of the entire sample of signals. In that case, bidders need not be concerned with the information they reveal in the event when they lose the auction, and thus bidding and auction revenue are unaffected by the royalty scheme. The sufficient statistic case has some prominence in the literature on common value auctions.

A further difference between the private and the common values case concerns the effect of revealing information by publishing either both the winning and the losing bids or only the winning bid. In the corresponding private values model it makes no difference whether the innovator publishes all bids or only the winning bid. This is due to the fact that, in equilibrium, the losing bid is only informative for the innovator who sets the royalty rate equal to the inferred cost reduction, but not for the competing firm who believes, in equilibrium, that the loser pays a "fair" royalty rate equal to his cost reduction. Whereas in the common value model, the losing bid is not only informative for the innovator in assessing the royalty rate he should charge, but in addition is also informative for the winning bidder in assessing his own cost reduction. ${ }^{2}$

In the common value case, both signaling effects - the effect on the royalty rate set by the innovator and the effect on the winner's assessment of his own cost reduction exert a downward pressure on equilibrium bids. This suggests that by publishing only the winning bid, as we will assume in the present paper, the innovator removes one adverse effect on equilibrium bids which contributes to increase his expected profit.

\footnotetext{
${ }^{2}$ Incidentally, this difference suggests a testable property of the common vs. private values case.
} 
Another important link to the literature concerns the specification of the common value assumption. In the literature two specifications are prominently used: the so called "mineral rights" model, first introduced by Wilson (1977), and the highly stylized "wallet auction" model introduced by Klemperer (1998). Recently, Goeree and Offerman (2003) suggested that the simple wallet auction specification carries the main qualitative features of a common value problem, while avoiding the technical complexity of the mineral rights model. In the present paper we follow this suggestion and introduce a generalized wallet auction model in which the highest signal is equally or more informative than the lowest signal. The main advantage of this proposed specification is that, unlike the standard wallet auction, it contains the well-known "sufficient statistic" example of the mineral rights model as a special case. That "sufficient statistic" example (Wilson, 1977, see Example 1) has been used repeatedly in the literature (see, for example Harstad and Levin, 1985) who show that in this case the Vickrey auction is dominance solvable.

Our main findings can be summarized as follows: 1) The equilibrium bid function is strictly monotone increasing if the reliability function is log-concave ("hazard rate monotonicity"). 2) Adding the royalty scheme adversely affects bidding, which lowers the innovator's revenue from the auction, except in the case when the signals is a sufficient statistic of the entire sample of signals. 3) We provide several further sufficient conditions for which the additional royalty income weighs more than that loss in auction revenue and illustrate the usefulness of these conditions with examples.

The paper is organized as follows: In Section 2 we present the model and introduce basic assumptions. In Section 3 we solve the oligopoly subgames that may occur if a bidder unilaterally deviates from the equilibrium bid, and then use the result to find the equilibrium bid function. Thereby, the second order conditions help us identify parameter restrictions to assure existence of a separating equilibrium. In Section 4 we state sufficient conditions for the profitability of the royalty scheme and illustrate the utility of these conditions with examples. In Section 5 we discuss our results and sketch some extensions. Some of the proofs and a full scale example are contained in the Appendix.

\section{Model}

An outsider innovator auctions a cost-reducing process innovation protected by a patent to a Cournot duopoly. The cost reduction induced by the innovation is independent of who uses it (common value case) and is unknown at the time of the auction. However, prior to the auction each firm draws an imperfect, private signal of the unknown cost reduction.

The innovator adopts the following mechanism: one license is auctioned to the highest bidder in a first-price auction, with the provision that the loser must sign a linear royalty contract. The winner pays his bid, while the loser pays a fixed royalty rate per output unit equal to the expected cost reduction inferred from the bids jointly observed by the loser and the auctioneer.

After the auction, the innovator publishes the winning (and perhaps also the losing) bid(s), and the firms then play a Cournot duopoly market game under incomplete information. 
Firms are subject to linear cost, $C_{i}\left(q_{i}\right)=c_{i} q_{i}$, and a concave inverse demand function, $P(Q), Q=q_{i}+q_{j}$, where $c_{i}<\lim _{Q \rightarrow 0}$, and the cost reduction induced by the innovation is non-drastic in the sense that its exclusive adoption cannot propel monopoly. The prior cost is $c$ and the expected cost after the innovation is equal to $c$ minus the expected cost reduction due to the innovation.

Inspired by the Klemperer (1998) and Goeree and Offerman (2003), we employ a simple "wallet auction" like specification of the common value cost reduction. There, bidders draw iid signals $X_{i}$, and the expected cost reduction is equal to a weighted average of the ordered signals:

$$
\theta\left(X_{(1)}, X_{(2)}\right)=t X_{(1)}+(1-t) X_{(2)}, \quad t \in[1 / 2,1],
$$

where $X_{(1)}>X_{(2)}$ denotes the order statistics of the sample of signals.

Each firm privately observes its signal but not that of the other firm. However, after the outcome of the auction is observed, firms may update their beliefs about their rival's signal, using Bayes' rule when it applies.

Signals are iid random variables, drawn from the probability distribution $F:[d, c] \rightarrow$ $[0,1]$ with positive density $f$ everywhere. The distribution function exhibits a logconcave reliability function, $1-F(x)$, which is equivalent to a strictly increasing hazard rate. This assumption rules out that $F$ has parts that are highly concave and is satisfied by many standard distribution functions (see the survey by Bagnoli and Bergstrom, 2005).

We assume that the support of $F$ is sufficiently bounded away from zero, i.e., $d$ is positive and sufficiently large. This implies that the innovation entails a positive expected cost reduction with probability one. As will become clear later on, this assumption is necessary to assure existence of a separating equilibrium. We mention that $d>0$ can be a property of the innovation technology but may also be the result of a positive reserve price.

We also mention that, unlike the original "wallet auction" model which assumes a simple average of signals, the above specification puts equal or more weight on the highest signal. This allows us to also capture the "sufficient statistic case" that figures prominently in the mineral rights model (see Wilson, 1977, Harstad and Levin, 1985). In that case, the highest signal is a sufficient statistic of the entire sample of signals, i.e. lower signals carry no additional information. Evidently, in our specification this case is a special obtained for $t=1$.

Recently, Goeree and Offerman (2003) argued that the "wallet specification" deserves wider use since it carries the essential qualitative features of the mineral rights model while avoiding the technical complexity of the latter. This is particularly true for the generalized wallet model specification introduced above.

\section{Equilibrium play with/without royalty scheme}

We now analyze the proposed mechanism assuming that only the winning bid is published, using the following methodology. First, we solve the oligopoly subgames that may occur if a player, say player 1 , who has drawn signal $x$, unilaterally deviates from 
the equilibrium bidding strategy and bids as if his signal were equal to $z \in[d, c]$ rather than $x$. Second, we use the working hypothesis that the bidding game has a unique symmetric and strictly increasing equilibrium, $\beta:[d, c] \rightarrow \Re$. This implies that the innovator, who observes both bids, can select the winner and set the royalty rate equal to the expected cost reduction inferred from observed bids, while bidders can only draw an inference from the observed winning bid. Of course, we will show that this working hypothesis confirms for the equilibrium that we find using this hypothesis.

\subsection{Equilibria of the oligopoly subgames}

Without loss of generality, consider bidder 1 who unilaterally deviates from the equilibrium bidding strategy and bids $\beta(z)$ even though he has drawn signal $x$ rather than $z$. In the continuation oligopoly game, bidder 1 faces the following subgames with positive probability, depending upon the relationship between his true signal, $x$, his pretended signal $z$, and the signal drawn by bidder $2, y$. We distinguish between the cases that occur due to "overbidding", $z>x$, and due to "underbidding", $z<x$, respectively. The purpose of the analysis is to compute the payoff function of firm $1, \Pi(x, z)$, which is needed in order to compute the equilibrium strategy $\beta$.

By construction, both bidders believe that their rival plays equilibrium, and therefore both take the signal revealed by bids at face value, as if it were the true signal. This implies immediately, that, if the royalty scheme is adopted, the winner believes that the loser pays the "fair" royalty rate equal to the expected cost reduction. In other words, the winner believes that the expected unit cost of the loser is equal to the prior unit cost $c$.

For convenience, denote firms' net expected unit cost by $c_{i}$, which is equal to $c-\theta$, if firm $i$ has won the auction, equal to $c_{i}=c$ if firm $i$ has the lost the auction and no royalty scheme is adopted, and equal to $c_{i}=c-\theta+r$ if firm $i$ lost the auction and the royalty scheme is adopted. Also denote firms' payoff function in the oligopoly game by $\pi_{i}\left(q_{i}, q_{j} ; c_{i}\right):=\left(P\left(q_{i}+q_{j}\right)-c_{i}\right) q_{i}, \quad i, j \in\{1,2\}, i \neq j$. From the above we know that the winning firm believes that the losing firm pays a "fair" royalty rate, if the royalty scheme is adopted. Therefore, the winning firm believes that the losing firm's effective unit cost is equal to $c$, regardless of whether the royalty scheme is adopted.

\subsubsection{Subgames that may occur by bidding above equilibrium: $z>x$}

Each Cournot oligopoly subgame is identified by the respective profile of units costs, $\left(c_{1}, c_{2}\right)$. Therefore, we denote these subgames by $\Gamma\left(c_{1}, c_{2}\right)$.

Case a): $\mathbf{z}>\mathbf{y} \quad$ In these events firm 1 won the auction. Firm 1 privately knows that its signal is $x$, not $z$.

To compute the equilibrium payoff of firm 1 in this case, one must first solve the game that firm 1 believes that firm 2 believes to be playing. We denote that game by $\Gamma_{a}$ (the subscript indicates the case).

As explained already, the winner believes that the loser pays a "fair" royalty rate; therefore, $c_{2}=c$. The loser, firm 2, believes that the highest signal is $z$ and firm 1 knows this. However, firm 1 does not know the signal observed by firm 2, it only 
knows that this signal is below $z$, and this fact is common knowledge. Therefore, firm 1 believes that firm 2 believes that $c_{1}=c-\theta(z, \mu(z))$, where $\mu(z)$ is the conditional expected value of the signal observed by firm 2 , in the eyes of firm 1 :

$$
\mu(z):=\int_{d}^{z} y \frac{d F(y)}{F(z)} .
$$

Thus, $\Gamma_{a}=\Gamma(c-\theta(z, \mu(z)), c)$.

Denote the equilibrium strategy of firm 2 of that game by $q_{2_{a}}(z)$. Firm 1 predicts that firm 2 plays this strategy. Since firm 1 privately knows that its true signal is equal to $x$ rather than $z$, it plays its best reply to $q_{2_{a}}(z)$.

When firm 1 computes its best reply to $q_{2_{a}}(z)$, it takes into account that it privately knows its true signal, $x$. Since the losing bid is not published, firm 1 only knows that the signal of firm 2 is below $z$, but it does not know whether its own signal is the highest. Using the information available to firm 1, it computes its expected cost reduction which is:

$$
\theta_{1}(x, z):=\int_{d}^{x} \theta(x, y) \frac{d F(y)}{F(z)}+\int_{x}^{z} \theta(y, x) \frac{d F(y)}{F(z)} .
$$

The best-reply of firm 1 to $q_{2_{a}}(z)$ is

$$
q_{1_{a}}(x, z)=\underset{q_{1}}{\arg \max } \pi_{1}\left(q_{1}, q_{2_{a}}(z) ; c-\theta_{1}(x, z)\right) .
$$

Therefore, in case a) with and without royalty scheme the equilibrium payoff of firm 1 is (the superscript $n$ indicates the case when "no" royalty scheme is used):

$$
\Pi_{a}(x, z)=\Pi_{a}^{n}(x, z)=\pi_{1}\left(q_{1_{a}}(x, z), q_{2_{a}}(z) ; c-\theta_{1}(x, z)\right) .
$$

Case b): $\mathbf{y}>\mathbf{z}$ In these events firm 2 won the auction, and both firms agree that the highest signal is $y$.

Firm 1 believes that firm 2 believes to play the oligopoly game: $\Gamma_{b}=\Gamma(c, c-\theta(y, \mu(y))$, with the equilibrium strategies $\left(q_{1_{b}}^{n}(y), q_{2_{b}}^{n}(y)\right)$.

Firm 1 has private information concerning its signal, but it can only take advantage of this private information if the royalty scheme is adopted.

If no royalty scheme is adopted, the best reply of firm 1 to $q_{2_{b}}^{n}(y)$ is simply $q_{1_{b}}^{n}(y)$. Therefore, in this case, the equilibrium payoff of firm 1 is

$$
\Pi_{b}^{n}(y)=\pi_{1}\left(q_{1_{b}}^{n}(y), q_{2_{b}}^{n}(y) ; c\right) .
$$

Whereas if the royalty scheme is adopted, firm 1 understands that its signaling strength $(z>x)$ backfired since it now has to pay an excessively high royalty rate equal to $\theta(y, z)$ that exceeds its true cost reduction, $\theta(y, x)$. The best reply of firm 1 to $q_{2_{b}}^{n}(y)$ becomes

$$
q_{1_{b}}(x, z, y)=\arg \max _{q_{1}} \pi_{1}\left(q_{1}, q_{2_{b}}^{n}(y) ; c-\theta(y, x)+\theta(y, z)\right) .
$$

Therefore, in case b) with royalty scheme, the equilibrium payoff of firm 1 is

$$
\Pi_{b}(x, z, y)=\pi_{1}\left(q_{1_{b}}(x, z, y), q_{2_{b}}^{n}(y) ; c-\theta(y, x)+\theta(y, z)\right) .
$$

Note, in case b) the result depends on whether the royalty scheme is adopted. 


\subsubsection{Subgames that may occur by bidding below equilibrium: $\mathrm{z}<\mathrm{x}$}

Case c): $\mathbf{z}>\mathbf{y}$ In these events firm 1 won the license auction. Firm 2 believes that the signal drawn by firm 1 is equal to $z$; firm 1 privately knows that its signal is $x>z$.

By reasoning analogous to the above, firm 1 believes that firm 2 believes to play the oligopoly game $\Gamma_{c}=\Gamma(c-\theta(z, \mu(z)), c)$. Denote the equilibrium strategy of firm 2 by $q_{2_{c}}(z)$. Firm 1 uses its private information, and plays its best reply to $q_{2_{c}}(z)$ :

$$
q_{1_{c}}(x, z)=\underset{q_{1}}{\arg \max } \pi_{1}\left(q_{1}, q_{2_{c}}(z) ; c-\theta(x, \mu(z))\right) .
$$

Therefore, in case c), with or without royalty scheme, the equilibrium payoff of firm 1 is

$$
\Pi_{c}(x, z)=\Pi_{c}^{n}(x, z)=\pi_{1}\left(q_{1_{c}}(x, z), q_{2_{c}}(z) ; c-\theta(x, \mu(z))\right) .
$$

Case d): $\mathbf{x}>\mathbf{y}>\mathbf{z}$ In these events firm 2 won the license auction. Firm 2 believes that the highest signal is $y$; firm 1 privately knows that the highest signal is $x$.

Firm 1 believes that firm 2 believes to play the oligopoly game $\Gamma_{d}=\Gamma(c, c-\theta(y, \mu(y))$, with the equilibrium strategies $\left(q_{1_{d}}^{n}(y), q_{2_{d}}^{n}(y)\right)$, regardless of whether the royalty scheme is adopted.

Therefore, in case d) without royalty scheme, the equilibrium is $\left(q_{1_{d}}^{n}(y), q_{2_{d}}^{n}(y)\right)$, and the equilibrium payoff of firm 1 is equal to

$$
\Pi_{d}^{n}(y)=\pi_{1}\left(q_{1_{d}}^{n}(y), q_{2_{d}}^{n}(y) ; c\right) .
$$

Whereas, if the royalty scheme is adopted, firm 1 plays its best reply to $q_{2_{d}}^{n}(y)$ which is:

$$
q_{1_{d}}(x, z, y)=\underset{q_{1}}{\arg \max } \pi_{1}\left(q_{1}, q_{2_{d}}^{n}(y) ; c-\theta(x, y)+\theta(y, z)\right) .
$$

Therefore, in case d) with royalty scheme the equilibrium payoff of firm 1 is

$$
\Pi_{d}(x, z, y)=\pi_{1}\left(q_{1_{d}}(x, z, y), q_{2_{d}}^{n}(y) ; c-\theta(x, y)+\theta(y, z)\right) .
$$

Case e): $\mathbf{y}>\mathbf{x}$ In these events firm 2 won the license auction; firm 2 believes that the highest signal is $y$.

Firm 1 believes that firm 2 believes to play the oligopoly game $\Gamma_{e}=\Gamma(c, c-\theta(y, \mu(y))=$ $\Gamma_{d}$, with the equilibrium strategies $\left(q_{1_{d}}^{n}(y), q_{2_{d}}^{n}(y)\right)$.

Therefore, in case e) without royalty scheme, the equilibrium is $\left(q_{1_{d}}^{n}(y), q_{2_{d}}^{n}(y)\right)$, and the equilibrium expected payoff of firm 1 is equal to $\Pi_{d}^{n}(y)$.

Whereas if the royalty scheme is adopted, firm 1 plays the strategy

$$
q_{1_{e}}(x, z, y)=\underset{q_{1}}{\arg \max } \pi_{1}\left(q_{1}, q_{2_{d}}^{n}(y) ; c-\theta(y, x)+\theta(y, z)\right) .
$$

Therefore, in case e) with royalty scheme the equilibrium payoff of firm 1 is

$$
\Pi_{e}(x, z, y)=\pi_{1}\left(q_{1_{e}}(x, z, y), q_{2_{d}}^{n}(y) ; c-\theta(y, x)+\theta(y, z)\right) .
$$


Payoff functions $\Pi(x, z) \quad$ Altogether, the expected oligopoly payoff of bidder 1 who has drawn signal $x$ but bids as if he had drawn signal $z$, while firm 2 plays equilibrium, is equal to $\Pi_{u p}$ for $z \geq x$ and $\Pi_{d n}$ for $z \leq x$, where:

$$
\begin{aligned}
& \Pi_{u p}(x, z)=\Pi_{a}(x, z) F(z)+\int_{z}^{c} \Pi_{b}(x, z, y) d F(y) \\
& \Pi_{d n}(x, z)=\Pi_{c}(x, z) F(z)+\int_{z}^{x} \Pi_{d}(x, z, y) d F(y)+\int_{x}^{c} \Pi_{e}(x, z, y) d F(y) .
\end{aligned}
$$

If no royalty scheme is adopted, $\Pi_{u p}^{n}(x, z)$ and $\Pi_{d n}^{n}(x, z)$ are obtained simply by replacing $\Pi_{b}(x, z, y)$ by $\Pi_{b}^{n}(y), \Pi_{d}(x, z, y)$ by $\Pi_{d}^{n}(y)$, and $\Pi_{e}(x, z, y)$ by $\Pi_{d}^{n}(y)$.

Outputs on the equilibrium path If bidders play the symmetric, strictly increasing equilibrium bidding strategy, the winner $W$ and the loser $L$ of the auction infer the signal drawn by the winner, $x_{W}$, the procurer infers the signals drawn by the winner and the loser, $\left(x_{W}, x_{L}\right)$, and firms play the oligopoly subgame $\Gamma^{*}=\Gamma\left(c-\theta\left(x_{W}, \mu\left(x_{W}\right), c\right)\right.$ with the equilibrium strategies $\left(q_{W}^{*}\left(x_{W}\right), q_{L}^{*}\left(x_{W}\right)\right)$.

And if the royalty scheme is adopted, the innovator earns the royalty income

$$
R^{*}\left(x_{W}, x_{L}\right)=\theta\left(x_{W}, x_{L}\right) q_{L}^{*}\left(x_{W}\right) .
$$

\subsection{Equilibrium bidding strategy}

Suppose bidder 1 has drawn signal $x$ but bids as if he had drawn signal $z \geq x .^{3}$

As a working hypothesis assume $\beta$ and $\beta_{n}$ are strictly increasing (which we will confirm later). Then, the bid functions with royalty scheme, $\beta$, must satisfy the equilibrium requirement that

$$
x=\arg \max _{z}\left(\Pi_{u p}(x, z)-\beta(z) F(z)\right), \forall x \in[d, c] .
$$

Similar conditions apply to $\beta_{n}$ after replacing $\Pi_{u p}$ by $\Pi_{u p}^{n}$.

Defining "value functions" $v, v_{n}$, denoting the "on the equilibrium path" oligopoly profits by $\Pi_{a}^{*}(x):=\left.\Pi_{a}(x, z)\right|_{z=x}, \Pi_{b}^{*}(x, y):=\left.\Pi_{b}(x, z, y)\right|_{z=x}$, and using the fact that $\left.\Pi_{b}^{*}(x, y)\right|_{y=x}=\left.\Pi_{b}^{n}(y)\right|_{y=x}$, the equilibrium requirements concerning $\beta$ resp. $\beta_{n}$ can be written in the form:

$$
\begin{aligned}
(\beta(x) F(x))^{\prime} & =v(x) f(x), \quad \text { resp. } \quad\left(\beta_{n}(x) F(x)\right)^{\prime}=v_{n}(x) f(x) \\
v(x): & =v_{n}(x)+\left.\int_{x}^{c} \partial_{z} \Pi_{b}(x, z, y)\right|_{z=x} \frac{f(y)}{f(x)} d y \\
v_{n}(x): & =\Pi_{a}^{*}(x)-\left.\Pi_{b}^{n}(y)\right|_{y=x}+\left.\partial_{z} \Pi_{a}(x, z)\right|_{z=x} \frac{F(x)}{f(x)} .
\end{aligned}
$$

Integrating the above differential equations gives

$$
\beta(x)=\int_{d}^{x} v(y) \frac{f(y)}{F(x)} d y, \quad \text { resp. } \quad \beta_{n}(x)=\int_{d}^{x} v_{n}(y) \frac{f(y)}{F(x)} d y .
$$

\footnotetext{
${ }^{3}$ Although the functions $\Pi_{u p}$ and $\Pi_{d n}$ differ, they yield the same differential equation. However, when we check the second-order conditions, we need to consider both branches of the payoff function $\Pi(x, z)$.
} 
Since the construction is based on the working hypothesis that $\beta$ and $\beta_{n}$ are strictly increasing, we now state a sufficient condition that confirms it and then also check whether the solution of the first-order conditions describes a global maximum.

Lemma 1. $\beta$ and $\beta_{n}$ are strictly increasing if the functions $v$ and $v_{n}$ are strictly increasing.

Proof. Differentiating (23) gives $\beta^{\prime}(x)=\frac{f(x)}{F(x)^{2}} \int_{d}^{x} v^{\prime}(y) F(y) d y \geq 0$ (the same relationship applies to $\beta_{n}$ and $v_{n}$ ).

If $\beta$ and $\beta_{n}$ are monotone increasing and if second order conditions are satisfied, it follows that:

Proposition 1. If $t=1$, the introduction of royalty contracts for losers does not affect bidding, whereas if $t<1$ it induces pointwise lower bids: $\beta(x)<\beta_{n}(x)$ for all $x$.

Proof. If $t=1$, the lower signal is uninformative both to the innovator and to the firm who has drawn the lower signal. Therefore, the royalty rate and the cost reduction of the loser are independent of the lower signal so that the loser's effective unit cost is equal to $c$. Therefore, $\left.\partial_{z} \Pi_{b}(x, z, y)\right|_{z=x}=0$, and thus $v(x)=v_{n}(x)$, which implies $\beta(x)=\beta_{n}(x)$. In other words, if $t=1$, adding the royalty scheme entails no loss in auction revenue.

Let $t<1$. The bid functions are fully determined by the "value" functions, $v, v_{n}$. By the envelope theorem and the facts that $q_{2_{b}}^{n}$ is only a function of the winner's signal, $y$, and, for $z=x$ (i.e. on the equilibrium path) the output $q_{1_{b}}$ is only a function of the winner's signal, $y$, one has

$$
\begin{aligned}
\left.\partial_{z} \Pi_{b}(x, z, y)\right|_{z=x} & =-\left.\left.\partial_{z} \theta(y, z)\right|_{z=x} q_{1_{b}}(x, z, y)\right|_{z=x} \\
& =-\left.(1-t) q_{1_{b}}(x, z, y)\right|_{z=x} \\
& =-(1-t) q_{L}^{*}(y)<0
\end{aligned}
$$

and hence, using (21), $v(x)<v_{n}(x), \beta(x)<\beta_{n}(x)$ for all $x$.

Proposition 2 (Monotonicity). The equilibrium bid function $\beta_{n}$, is strictly increasing, and the equilibrium bid function $\beta$ is strictly increasing if the reliability function is log-concave.

Proof. By Lemma (1) the bid functions $\beta_{n}, \beta$ are strictly increasing if $v_{n}$ and $v-v_{n}$ are strictly increasing. We confirm the latter.

Evidently, $\Pi_{a}^{*}(x)$ is strictly increasing for two reasons: 1) because a higher $x$ lowers the winner's unit cost and thus increases his profit margin, and 2) as the loser infers the higher cost reduction of the winner from the winner's equilibrium bid, the loser reduces his output.

Similarly, $\left.\Pi_{b}^{*}(x, y)\right|_{y=x}$ is strictly decreasing in $x$ for two reasons: 1$)$ on the equilibrium path, i.e. for $z=x$, the loser's unit costs is independent of signals; 2) as the signal of the rival, $y$, is increased, the rival increases his output, which reduces the loser's profit. 
Therefore, $v_{n}(x):=\Pi_{a}^{*}(x)-\left.\Pi_{b}^{*}(x, y)\right|_{y=x}$ and hence $\beta_{n}$ is strictly increasing.

To show that $v-v_{n}$ is strictly increasing, we compute the derivative of $v(x)-v_{n}(x)$, which is assessed as follows:

$$
\begin{aligned}
\left(v(x)-v_{n}(x)\right)^{\prime}= & \frac{d}{d x}\left(\left.\frac{1}{f(x)} \int_{x}^{c} \partial_{z} \Pi_{b}(x, z, y)\right|_{z=x} d F(y)\right) \\
= & \frac{1}{f(x)} \int_{x}^{c}\left(\left.\partial_{z x} \Pi_{b}(x, z, y)\right|_{z=x}+\left.\partial_{z z} \Pi_{b}(x, z, y)\right|_{z=x}\right) d F(y) \\
& -\left.\partial_{z} \Pi_{b}(x, z, y)\right|_{z=y=x}-\left.\frac{f^{\prime}(x)}{f(x)^{2}} \int_{x}^{c} \partial_{z} \Pi_{b}(x, z, y)\right|_{z=x} d F(y) \\
= & -\left.\partial_{z} \Pi_{b}(x, z, y)\right|_{z=y=x}-\left.\frac{f^{\prime}(x)}{f(x)^{2}} \int_{x}^{c} \partial_{z} \Pi_{b}(x, z, y)\right|_{z=x} d F(y) \quad \text { (step a) } \\
> & -\left.\partial_{z} \Pi_{b}(x, z, y)\right|_{z=y=x}+\left.\frac{1}{1-F(x)} \int_{x}^{c} \partial_{z} \Pi_{b}(x, z, y)\right|_{z=x} d F(y) \quad \text { (step b) } \\
> & -\left.\partial_{z} \Pi_{b}(x, z, y)\right|_{z=y=x}+\left.\frac{1}{1-F(x)} \int_{x}^{c} \partial_{z} \Pi_{b}(x, z, y)\right|_{z=y=x} d F(y) \quad \text { (step c) } \\
= & -\left.\partial_{z} \Pi_{b}(x, z, y)\right|_{z=y=x}+\left.\partial_{z} \Pi_{b}(x, z, y)\right|_{z=y=x}=0 .
\end{aligned}
$$

The different steps in this assessment are explained as follows.

Step a) follows from the fact that

$$
\left.\partial_{z x} \Pi_{b}(x, z, y)\right|_{z=x}+\left.\partial_{z z} \Pi_{b}(x, z, y)\right|_{z=x} \equiv 0,
$$

as we show in Appendix A.1.

Step b) follows from the assumed log-concavity of the reliability function, which implies that $f^{\prime}(x)>-f(x)^{2} /(1-F(x))$, together with the fact that $\left.\partial_{z} \Pi_{b}(x, z, y)\right|_{z=x}<0$.

Step c) follows from the fact that $\left.\partial_{z} \Pi_{b}(x, z, y)\right|_{z=x}=-\left.\left.\partial_{z} \theta(y, z)\right|_{z=x} q_{1_{b}}(x, z, y)\right|_{z=x}$, by (24), which is monotone increasing in $y$ since $\theta$ is linear in $z$, and $q_{1_{b}}(x, z, y)$ is decreasing in $y$.

Having established sufficient conditions for the monotonicity of the bid functions that solves the first-order conditions of a maximum, we now state conditions for which these solutions yield a global maximum. For this purpose we examine whether bidders' payoff function is pseudoncave in the deviation variable $z$. Since the oligopoly equilibrium payoff function differs for upward $(z \geq x)$ and for downward deviations $(z \leq x)$, we need to examine the pseudoconcavity for both kinds of deviations, using the respective function $\bar{\Pi}_{u p}(x, z):=\Pi_{u p}(x, z)-\beta(z) F(z)$ and $\bar{\Pi}_{d n}(x, z):=\Pi_{d n}(x, z)-\beta(z) F(z)$.

The payoff function is pseudoconcave in $z$ if $\bar{\Pi}_{u p}(x, z)$ is decreasing in $z$ for all $z \geq x$ and if $\bar{\Pi}_{d n}(x, z)$ is increasing in $z$ for all $z \leq x$. Evidently, these conditions assure that a stationary point is a global maximum. A sufficient condition for pseudoconcavity is that the cross derivatives, $\partial_{z x} \bar{\Pi}_{u p}, \partial_{z x} \bar{\Pi}_{d n}$, are positive, since these assumptions imply:

$$
z<x \Rightarrow \partial_{z} \bar{\Pi}_{d n}(x, z)>\partial_{z} \bar{\Pi}_{d n}(z, z)=0, \quad z>x \Rightarrow \partial_{z} \bar{\Pi}_{u p}(x, z)<\partial_{z} \bar{\Pi}_{u p}(x, x)=0 .
$$

We will identify conditions under which this sufficient condition is satisfied. 
Lemma 2 (Second order conditions). 1) The constructed equilibrium bid function $\beta_{n}$, is a global maximizer of bidders' payoff function $\bar{\Pi}^{n}(x, z)$.

2) The constructed equilibrium bid function $\beta$, is a global maximizer of bidders' payoff function $\bar{\Pi}(x, z)$ if $d$ is positive and sufficiently large and if $t$ is sufficiently large.

Proof. We show in Appendix A.2 that the cross derivative of bidders' payoff function $\bar{\Pi}^{n}(x, z)$ is positive everywhere. That function is equal to $\bar{\Pi}_{u p}^{n}(x, z)$ for $z \geq x$ and equal to $\bar{\Pi}_{d n}^{n}(x, z)$ for $\left.z \leq x\right)$. Therefore, that function is pseudoconcave. And we show in Appendix A.3 that $\partial_{z x} \bar{\Pi}(x, z) \geq 0$ if the stipulated conditions concerning $d$ and $t$ are satisfied.

If the royalty scheme is adopted, the second order conditions are only satisfied if the parameter $t$ is sufficiently high, i.e. if the low signal has relatively small weight in determining the expected value of the cost reduction) or if $d$ is positive and sufficiently large (i.e. if signals are sufficiently bounded above zero). The intuitive reason for this can be explained as follows.

Suppose bidder 1 has drawn a "low" signal and bidder 2 bids according to the constructed bid function $\beta$. Then, bidder 1 should contemplate to bid the minimum, $\beta(d)$, i.e., bid as if his signal were equal to the lowest possible value from the signal support, because due to his low signal he tends to lose the auction anyway, but gets the innovation in exchange for paying the lowest possible royalty rate, $r=\theta(y, d)$. This large scale deviating bid can significantly increase the profit of bidder 1 if $t$ is sufficiently small and $d$ is sufficiently close to zero. Whereas if $t$ or $d$ is sufficiently high, the royalty rate cannot be lowered significantly by bidding the minimum, which then makes it not worthwhile to forego the chance to win the auction, no matter how small that chance may be. ${ }^{4}$

\section{Innovator's expected equilibrium profit}

We now compute the innovator's expected profits, with and without royalty scheme, $G, G^{n}$, and assess the profitability of the royalty scheme.

The equilibrium auction revenue is equal to the highest bid and the innovator's licensing income is equal to the equilibrium royalty income paid by the loser, $\theta(x, y) q_{L}^{*}(y, x)$.

\footnotetext{
${ }^{4}$ Note, in the private values case, the benefit from such a large scale deviation is considerably more pronounced, because there a bidder may get the innovation for free by bidding zero. This is why in the private values case, employing a sufficiently high reserve price is crucial in that case (as shown in Fan et al., 2009b).
} 
Therefore, one finds: ${ }^{5}$

$$
\begin{aligned}
G_{n}= & E\left(\beta_{n}\left(X_{(1)}\right)\right), \quad G=E\left(\beta\left(X_{(1)}\right)\right)+E\left(\theta\left(X_{(1)}, X_{(2)}\right) q_{L}^{*}\left(X_{(1)}\right)\right) \\
\Delta:=G-G_{n}= & E\left(\beta\left(X_{(1)}\right)-\beta_{n}\left(X_{(1)}\right)\right)+E\left(\theta\left(X_{(1)}, \mu\left(X_{(1)}\right)\right) q_{L}^{*}\left(X_{(1)}\right)\right) \\
= & \left.2 \int_{d}^{c} \int_{d}^{x} \int_{y}^{c} \partial_{z} \Pi_{b}(y, z, s)\right|_{z=y} d F(s) d y d F(x) \\
& +2 \int_{d}^{c} \theta(x, \mu(x)) q_{L}^{*}(x) F(x) d F(x) \\
= & -2(1-t) \int_{d}^{c} \int_{d}^{x} \int_{y}^{c} q_{L}^{*}(s) d F(s) d y d F(x) \\
& +2 \int_{d}^{c} \theta(x, \mu(x)) q_{L}^{*}(x) F(x) d F(x)
\end{aligned}
$$

We now state sufficient conditions for the superiority of the royalty scheme and then show how these conditions can be used to quickly check various examples.

Proposition 3 (Superiority of Royalty Scheme). The royalty scheme increases the innovator's expected profit, $\Delta>0$, if at least one of the following conditions holds, where $\bar{q}_{L}^{*}:=E\left(q_{L}^{*}(X)\right)$ :

$$
\begin{gathered}
d \geq \frac{1-t}{2-t} E(X) \\
\operatorname{Cov}\left(\theta(X, \mu(X)), q_{L}^{*}(X)\right) \geq-\bar{q}_{L}^{*} E((2 t-1) X+(1-t)(\mu(X)+d)), .
\end{gathered}
$$

Proof. The proof is in Appendix A.4.

The first sufficient condition (25) is particularly easy to apply and is independent of the form of the inverse demand function. Since $(1-t) /(2-t)$ is a decreasing function, ranging from zero at $t=1$ to $1 / 3$ at $t=1 / 2$, this condition becomes less restrictive as $t$ is increased towards 1. Specifically, the condition is satisfied for all $t$ if $d \geq 1 / 3 E(X)$. And since $E(X)<c$, it is satisfied for all $t$ if $d \geq c / 3$.

The second sufficient condition (26) can be used to identify the values of the parameter $t$ for which the royalty scheme is superior. Suppose inverse demand is linear, $P(\cdot)=1-\left(q_{1}+q_{2}\right)$, so that $q_{L}^{*}(x)=\left(1-2 c_{L}+c_{W}\right) / 3=(1-c-\theta(x, \mu(x))) / 3$, and signals are uniformly distributed. Then, condition (26) is an inequality concerning a second order polynomial with coefficients $\alpha_{0}, \alpha_{1}, \alpha_{2}:^{6}$

$$
\gamma(t):=\alpha_{0}+\alpha_{1} t+\alpha_{2} t^{2} \geq 0, \quad \text { where } \quad \alpha_{2}:=-\frac{5}{36}(c-d)^{2}<0 .
$$

Since $\gamma$ is concave in $t$ and $\gamma(1 / 2), \gamma(1)>0$, it follows immediately that $\Delta>0$ for all $t \geq 1 / 2$.

\footnotetext{
${ }^{5}$ The c.d.f. of the largest order statistic is $F(x)^{2}$ and the joint density of the two highest order statistics is equal to $2 f(x) f(y)$.

${ }^{6}$ Also: $\alpha_{0}=1 / 36\left(c(13 d-c)-2 c^{2}+(15-23 d) d\right), \alpha_{1}=1 / 36\left(5 c^{2}+c(9-25 d)+d(20 d-9)\right)$.
} 


\section{Discussion}

In the present paper we reconsider the licensing of a common value process innovation to a Cournot duopoly under incomplete information assuming firms observe imperfect private signals prior to the license auction. Unlike the previous literature, we assume that the innovator combines a restrictive license auction with a mandatory royalty contract for those who lose the auction. The addition of this royalty scheme gives rise to a dual signaling problem where the winning bid signals the winner's private information to rival bidder as well as to the innovator, and the losing bid exclusively signals the loser's private information to the innovator. The losing bidders use the information revealed by the winning bid to assess the winner's expected cost reduction, and the innovator uses the information revealed by bids to set the royalty rate the losers have to pay. Therefore, signalling strength, by bidding as if one had received a more favorable signal concerning the cost reduction, pays off in the event when one wins the auction but backfires and raises the own unit cost in the event when one loses the auction.

Our main finding is that the bidding game has a monotone equilibrium if the distribution of signals exhibits hazard monotonicity. Adding the royalty scheme does not affect bidding if the highest signal is a sufficient statistic of the sample of signals ("sufficient statistic" case). Otherwise, adding the royalty scheme reduces bidding pointwise, although the thus induced loss in auction revenue is more than compensated by the additional royalty income.

Compared to the private values model, the case for adding the royalty scheme is stronger. Also, revelation of the losing bid (in addition to the winning bid) affects bidding in the common value but not in the private values case, which suggests a testable property of the common vs. private value paradigm.

\section{A Appendix}

\section{A.1 Proof of Step a) in Proposition 2}

Proof. By definition, $q_{1_{b}}(x, z, y)$ satisfies the first-order condition of the best-reply problem (7) for all signals, which yields the identity in $z, x, y$ :

$$
P^{\prime}\left(q_{1_{b}}(x, z, y)+q_{2_{b}}(y)\right) q_{1_{b}}(x, z, y)+P(\cdot)-c+\theta(y, x)-\theta(y, z) \equiv 0 .
$$

Differentiating w.r.t. $z$ resp. $x$ gives:

$$
\begin{aligned}
& \left(2 P^{\prime}(\cdot)+P^{\prime \prime}(\cdot) q_{1_{b}}(\cdot)\right) \partial_{z} q_{1_{b}}(\cdot)-\partial_{z} \theta(y, z)=0 \\
& \left(2 P^{\prime}(\cdot)+P^{\prime \prime}(\cdot) q_{1_{b}}(\cdot)\right) \partial_{x} q_{1_{b}}(\cdot)+\partial_{x} \theta(y, x)=0 .
\end{aligned}
$$

Adding the two equations and setting $z=x$ gives

$$
\left(2 P^{\prime}(\cdot)+P^{\prime \prime}(\cdot) q_{1_{b}}(\cdot)\right)\left(\partial_{x} q_{1_{b}}(\cdot)+\partial_{z} q_{1_{b}}(\cdot)\right)=0 .
$$

Since inverse demand is decreasing and concave, $\left(2 P^{\prime}(\cdot)+P^{\prime \prime}(\cdot) q_{1_{b}}(\cdot)\right)<0$; hence, $\partial_{x} q_{1_{b}}(\cdot)+\partial_{z} q_{1_{b}}(\cdot)=0$. Using this result together with (24) and the fact that $\theta$ is a 
linear function, we conclude:

$$
\begin{aligned}
\left.\partial_{z x} \Pi_{b}(x, z, y)\right|_{z=x}+\left.\partial_{z z} \Pi_{b}(x, z, y)\right|_{z=x} & =-\left.\partial_{z} \theta(y, z)\right|_{z=x}\left(\partial_{x} q_{1_{b}}(\cdot)+\partial_{z} q_{1_{b}}(\cdot)\right) \\
& =0,
\end{aligned}
$$

as asserted.

\section{A.2 Proof of Lemma 2, Part 1)}

1) We show that $\partial_{z x} \bar{\Pi}_{u p}^{n}(x, z) \geq 0$, as follows:

$$
\begin{aligned}
\partial_{z x} \bar{\Pi}_{u p}^{n}(x, z) & =\partial_{z x} \Pi_{u p}^{n}(x, z) \\
& =\partial_{x}\left(\partial_{z} \Pi_{a}(x, z) F(z)+\left(\Pi_{a}(x, z)-\left.\Pi_{b}^{n}(y)\right|_{y=z}\right) f(z)\right) \\
& =F(z) \partial_{z x} \Pi_{a}(x, z)+f(z) \partial_{x} \Pi_{a}(x, z) .
\end{aligned}
$$

Using the envelope theorem and the fact that $q_{2_{a}}$ is independent of $x$, one has

$$
\partial_{x} \Pi_{a}(x, z)=q_{1_{a}}(x, z) \partial_{x} \theta_{1}(x, z) .
$$

Note that

$$
\partial_{x} \theta_{1}(x, z)=(2 t-1) \frac{F(x)}{F(z)}+1-t>0 \quad \text { since } t>1 / 2 .
$$

Therefore,

$$
\begin{aligned}
\partial_{z x} \Pi_{a}(x, z) & =\partial_{z} q_{1_{a}}(x, z) \partial_{x} \theta_{1}(x, z)+q_{1_{a}}(x, z) \partial_{z x} \theta_{1}(x, z) \\
& >q_{1_{a}}(x, z) \partial_{z x} \theta_{1}(x, z) \quad \text { (as explained below) } \\
& =-q_{1_{a}}(x, z)(2 t-1) \frac{F(x) f(z)}{F(z)^{2}}
\end{aligned}
$$

where the inequality follows from (27) together with the fact that $\partial_{z} q_{1_{a}}(x, z)>0$ (the loser reduces his output when the winner has signalled strength, and the winner takes advantage of it by increasing his own output).

Combining the above, gives

$$
\begin{aligned}
\partial_{z x} \bar{\Pi}_{u p}^{n}(x, z) & >q_{1_{a}}(x, z) f(z)\left(-(2 t-1) \frac{F(x)}{F(z)}+(2 t-1) \frac{F(x)}{F(z)}+1-t\right) \\
& =q_{1_{a}}(x, z) f(z)(1-t)>0 .
\end{aligned}
$$

2) Similarly, we show that $\partial_{z x} \bar{\Pi}_{d n}^{n}(x, z) \geq 0$, as follows:

$$
\partial_{z x} \bar{\Pi}_{d n}^{n}(x, z)=F(z) \partial_{z x} \Pi_{c}(x, z)+f(z) \partial_{x} \Pi_{c}(x, z) .
$$

Using the envelope theorem and the fact that $q_{2_{c}}$ is independent of $x$, one has

$$
\begin{aligned}
\partial_{x} \Pi_{c}(x, z) & =q_{1_{c}}(x, z) \partial_{x} \theta(x, \mu(z)) \\
& =q_{1_{c}}(x, z) t>0 .
\end{aligned}
$$

Therefore,

$$
\partial_{z x} \Pi_{c}(x, z)=t \partial_{z} q_{1_{c}}(x, z)>0 .
$$

Combining the above confirms that $\partial_{z x} \bar{\Pi}_{d n}^{n}(x, z)>0$ everywhere. 


\section{A.3 Proof of Lemma 2, Part 2)}

By definition of $\Pi_{u p}, \Pi_{u p}^{n}$ one has

$$
\Pi_{u p}(x, z)=\Pi_{u p}^{n}(x, z)+\int_{z}^{c}\left(\Pi_{b}(x, z, y)-\Pi_{b}^{n}(y)\right) d F(y) .
$$

Therefore, using $\Pi_{u p}^{n}$ and the fact that $\partial_{x} \Pi_{b}(x, z, y)=(1-t) q_{1_{b}}(x, z, y)$,

$$
\begin{aligned}
\partial_{z x} \bar{\Pi}_{u p}(x, z)= & \partial_{z x} \Pi_{u p}(x, z) \\
= & \partial_{z}\left(\partial_{x} \Pi_{u p}(x, z)\right) \\
= & \partial_{z}\left(\partial_{x} \Pi_{u p}^{n}(x, z)+\int_{z}^{c}\left(\partial_{x} \Pi_{b}(x, z, y)-\partial_{x} \Pi_{b}^{n}(y)\right) d F(y)\right) \\
= & \partial_{z}\left(\partial_{x} \Pi_{u p}^{n}(x, z)+(1-t) \int_{z}^{c} \partial_{x} q_{1_{b}}(x, z, y) d F(y)\right) \\
= & \partial_{z x} \Pi_{u p}^{n}(x, z)+(1-t)\left(\int_{z}^{c} \partial_{z} q_{1_{b}}(x, z, y) d F(y)-q_{1_{b}}(x, z, z) f(z)\right) \\
= & (1-t) q_{1_{a}}(x, z) f(z)+\left((2 t-1) \frac{F(x)}{F(z)}+1-t\right) F(z) \partial_{z} q_{1_{a}}(x, z) \\
& -(1-t) q_{1_{b}}(x, z, z) f(z)+(1-t) \int_{z}^{c} \partial_{z} q_{1_{b}}(x, z, y) d F(y) \\
= & (1-t) f(z)\left(q_{1_{a}}(x, z)-q_{1_{b}}(x, z, z)\right)+\left((2 t-1) \frac{F(x)}{F(z)}+1-t\right) F(z) \partial_{z} q_{1_{a}}(x, z) \\
& +(1-t)^{2} \int_{z}^{c} \frac{1}{2 P^{\prime}+P^{\prime \prime} q_{1_{b}}} d F(y)
\end{aligned}
$$

To explain the last step, which involves substituting $\partial_{z} q_{1_{b}}(\cdot)$, recall the first-order condition concerning $q_{1_{b}}$ :

$$
P\left(q_{1_{b}}(x, z, y)+q_{2_{b}}(y)\right)+P^{\prime}(\cdot) q_{1_{b}}(\cdot)-c+\theta(y, x)-\theta(y, z)=0 .
$$

Differentiating w.r.t. $z$ gives

$$
\partial_{z} q_{1_{b}}(\cdot)=\frac{1-t}{2 P^{\prime}+P^{\prime \prime}(\cdot) q_{1_{b}}(\cdot)} .
$$

Obviously, if $t=1$ the above cross derivative is positive. Whereas if $t<1$, it cannot be positive everywhere for $d$ close to or equal to zero. Because if $d$ is close to zero, one finds for small $x, z$ with $z \geq x$, that the first two terms in the last equation vanish, whereas the last term is negative, so that $\partial_{z x} \bar{\Pi}_{u p}(x, z)<0$.

However, if $d$ is positive and sufficiently large, the above cross derivative is positive for all sufficiently large $t$. Because, in that case the effect of an upward deviation on the royalty rate becomes insignificant, while the output difference between winner and loser does not vanish as $t$ is increased towards 1 . This indicates that the second order conditions is satisfied if $d$ is positive and sufficiently large and $t$ is sufficiently close to 1. 
Next consider downward deviations, $z \leq x$ and the associated payoff function $\Pi_{d n}(x, z)$. By definition of $\Pi_{d n}, \Pi_{d n}^{n}$ one has

$$
\Pi_{d n}(x, z)=\Pi_{d n}^{n}(x, z)-\int_{z}^{c} \Pi_{d}^{n}(y) d F(y)+\int_{z}^{x} \Pi_{d}(x, z, y) d F(y)+\int_{x}^{c} \Pi_{e}^{n}(x, z, y) d F(y) .
$$

Therefore, using $\Pi_{d n}^{n}$ and the facts that $\partial_{x} \Pi_{d}(x, z, y)=t q_{1_{d}}(x, z, y)$, and $\partial_{x} \Pi_{e}(x, z, y)=$ $(1-t) q_{1_{e}}(x, z, y)$, one has

$$
\begin{aligned}
\partial_{z x} \bar{\Pi}_{d n}(x, z)= & \partial_{z x} \Pi_{d n}(x, z) \\
= & \partial_{z}\left(\partial_{x} \Pi_{d n}(x, z)\right) \\
= & \partial_{z}\left(\partial_{x} \Pi_{d n}^{n}(x, z)+\int_{z}^{x} \partial_{x} \Pi_{d}(x, z, y) d F(y)+\int_{x}^{c} \partial_{x} \Pi_{e}(x, z, y) d F(y)\right) \\
= & \partial_{z x} \Pi_{d n}^{n}(x, z)-t q_{1_{d}}(x, z, z) f(z)+t \int_{z}^{x} \partial_{z} q_{1_{d}}(x, z, y) d F(y) \\
& +(1-t) \int_{x}^{c} \partial_{z} q_{1_{e}}(x, z, y) d F(y) \\
= & t F(z) \partial_{z} q_{1_{c}}(x, z)+t q_{1_{c}}(x, z) f(z)-t q_{1_{d}}(x, z, z) f(z) \\
& +t \int_{z}^{x} \partial_{z} q_{1_{d}}(x, z, y) d F(y)+(1-t) \int_{x}^{c} \partial_{z} q_{1_{e}}(x, z, y) d F(y) \\
= & t F(z) \partial_{z} q_{1_{c}}(x, z)+t f(z)\left(q_{1_{c}}(x, z)-q_{1_{d}}(x, z, z)\right) \\
& +(1-t)\left(\int_{z}^{x} \frac{t}{2 P^{\prime}+P^{\prime \prime} q_{1_{d}}} d F(y)+\int_{x}^{c} \frac{1-t}{2 P^{\prime}+P^{\prime \prime} q_{1_{e}}} d F(y)\right) .
\end{aligned}
$$

To explain the last step, which involves substituting $\partial_{z} q_{1_{d}}(\cdot)$ and $\partial_{z} q_{1_{e}}(\cdot)$, recall the first-order condition concerning $q_{1_{d}}$ :

$$
P\left(q_{1_{d}}(x, z, y)+q_{2_{d}}(y)\right)+P^{\prime}(\cdot) q_{1_{d}}(\cdot)-c+\theta(x, y)-\theta(y, z)=0 .
$$

Differentiating w.r.t. $z$ gives

$$
\partial_{z} q_{1_{d}}(\cdot)=\frac{1-t}{2 P^{\prime}+P^{\prime \prime}(\cdot) q_{1_{d}}(\cdot)} .
$$

Similarly,

$$
\partial_{z} q_{1_{e}}(\cdot)=\frac{1-t}{2 P^{\prime}+P^{\prime \prime}(\cdot) q_{1_{e}}(\cdot)} .
$$

Obviously, if $t=1$ the above cross derivative is positive since $\partial_{z} q_{1_{c}}(x, z)>0$ and $q_{1_{c}}(x, z)-q_{1_{d}}(x, z, z)>0$. Whereas, if $t<1$, it cannot be positive everywhere if $d$ is close to or equal to zero. Because in that case, one finds for small $x, z$ with $x \geq z$, that the first two terms in the last equation vanish, and the last term is negative, so that $\partial_{z x} \bar{\Pi}_{d n}(x, z)<0$.

However, if $d$ is positive and sufficiently large, the cross derivative is positive for all sufficiently large $t$. Because, then the effect of a downward deviation on the royalty rate becomes insignificant, while the output difference between winner (case c) and loser (cases $\mathrm{d}$ and e) do not vanish as $t$ is increased towards 1 . This indicates that the second order conditions is satisfied if $d$ is positive and sufficiently large and $t$ is sufficiently close to 1 . 


\section{A.4 Proof of Proposition 3}

1) Proof of condition (25):

$$
\begin{aligned}
\frac{1}{2} \Delta & =-(1-t) \int_{d}^{c} \int_{d}^{x} \int_{y}^{c} q_{L}^{*}(s) d F(s) d y d F(x)+\int_{d}^{c} \theta(x, \mu(x)) q_{L}^{*}(x) d F(x) \\
& >-(1-t) \int_{d}^{c} \int_{d}^{x} \int_{d}^{c} q_{L}^{*}(s) d F(s) d y d F(x)+\int_{d}^{c} \theta(x, \mu(x)) q_{L}^{*}(x) d F(x) \\
& =-(1-t) \int_{d}^{c} \int_{d}^{x} \bar{q}_{L}^{*} d y d F(x)+\int_{d}^{c} \theta(x, \mu(x)) q_{L}^{*}(x) d F(x) \\
& \geq-(1-t) \bar{q}_{L}^{*} \int_{d}^{c} \int_{d}^{x} 1 d y d F(x)+d \int_{d}^{c} q_{L}^{*}(x) d F(x) \\
& =-(1-t) \bar{q}_{L}^{*} \int_{d}^{c}(x-d) d F(x)+d \bar{q}_{L}^{*} \\
& =-(1-t) \bar{q}_{L}^{*} E(X-d)+d \bar{q}_{L}^{*} \\
& =\bar{q}_{L}^{*}[(2-t) d-(1-t) E(X)] \\
& \geq 0, \text { if } d \geq \frac{1-t}{2-t} E(X) .
\end{aligned}
$$

2) Proof of condition (26):

$$
\begin{aligned}
\frac{1}{2} \Delta & =-(1-t) \int_{d}^{c} \int_{d}^{x} \int_{y}^{c} q_{L}^{*}(s) d F(s) d y d F(x)+\int_{d}^{c} \theta(x, \mu(x)) q_{L}^{*}(x) d F(x) \\
> & -(1-t) \int_{d}^{c} \int_{d}^{x} \int_{d}^{c} q_{L}^{*}(s) d F(s) d y d F(x)+\int_{d}^{c} \theta(x, \mu(x)) q_{L}^{*}(x) d F(x) \\
& =-(1-t) \int_{d}^{c} \int_{d}^{x} \bar{q}_{L}^{*} d y d F(x)+\int_{d}^{c} \theta(x, \mu(x)) q_{L}^{*}(x) d F(x) \\
& =-(1-t) \bar{q}_{L}^{*} \int_{d}^{c} \int_{d}^{x} 1 d y d F(x)+\int_{d}^{c} \theta(x, \mu(x)) q_{L}^{*}(x) d F(x) \\
& =-(1-t) \bar{q}_{L}^{*} \int_{d}^{c}(x-d) d F(x)+\int_{d}^{c} \theta(x, \mu(x)) q_{L}^{*}(x) d F(x) \\
& =-(1-t) \bar{q}_{L}^{*} \int_{d}^{c}(x-d) d F(x)+\left[\bar{q}_{L}^{*} \int_{d}^{c} \theta(x, \mu(x)) d F(x)+\operatorname{Cov}\left(\theta(x, \mu(x)), q_{L}^{*}(x)\right)\right] \\
& =\bar{q}_{L}^{*} E(\theta(x, \mu(x))-(1-t)(x-d))+\operatorname{Cov}\left(\theta(x, \mu(x)), q_{L}^{*}(x)\right) \\
& =\bar{q}_{L}^{*} E(t x+(1-t) \mu(x)-(1-t)(x-d))+\operatorname{Cov}\left(\theta(x, \mu(x)), q_{L}^{*}(x)\right) \\
& =\bar{q}_{L}^{*} E((2 t-1) x+(1-t)(\mu(x)+d))+\operatorname{Cov}\left(\theta(x, \mu(x)), q_{L}^{*}(x)\right) .
\end{aligned}
$$

\section{A.5 Model with linear demand and uniformly distributed signals}

As an illustration of our general analysis we sketch a full scale example that assumes linear demand, $P\left(q_{1}, q_{2}\right):=1-q_{1}-q_{2}$, uniformly distributed signals, and $c<1 / 2$ (non-drastic innovation). As one can easily confirm, in this case one has

$$
\begin{aligned}
\Pi_{u p}^{n}= & \frac{1}{432(c-d)}\left[\frac{4}{1+t}\left\{((3+t) c+(1-t) d-2)^{3}-((1+t) z+(1-t) d-2(1-c))^{3}\right\}\right. \\
& \left.+\frac{3}{z-d}\left\{4(1-c) z+(1+4 t) z^{2}+6(1-t) x z+3(2 t-1) x^{2}-4(1-t) d^{2}\right\}^{2}\right]
\end{aligned}
$$




$$
\begin{aligned}
\Pi_{d n}^{n}= & \frac{1}{108(c-d)}\left[\frac{1}{1+t}\left\{((3+t) c+(1-t) d-2)^{3}-((1+t) z+(1-t) d-2(1-c))^{3}\right\}\right. \\
& \left.+3(z-d)\{2(1-c)+2(1-t) d+3 t x+(2-t) z\}^{2}\right] \\
\Pi_{u p}= & \frac{1}{432(c-d)}\left[\frac { 4 } { 1 + t } \left\{((3+t) c+(1-t)(3(z-x)+d)-2)^{3}\right.\right. \\
& \left.-(2(2-t) z+(1-t)(d-3 x)-2(1-c))^{3}\right\} \\
& +\frac{3}{z-d}\left\{4(1-c)(z-d)+(1+4 t) z^{2}-2 t(3 x+z) d\right. \\
& \left.\left.+2(1-t)\left(3 x z-2 d^{2}\right)+3(2 t-1) x^{2}\right\}^{2}\right] \\
\Pi_{d n}= & \frac{1}{108(c-d)}\left[\frac { 1 } { 1 + t } \left\{((3+t) c+(1-t)(3(z-x)+d)-2)^{3}\right.\right. \\
& \left.-(2(2 t-1) x+(1-t)(3 z+d)-2(1-c))^{3}\right\} \\
& +\frac{1}{7 t-2}\left\{(2(2 t-1) x+(1-t)(3 z+d)-2(1-c))^{3}\right. \\
& \left.-((4 t+1) z-3 t x+(1-t) d-2(1-c))^{3}\right\} \\
& \left.+3(z-d)\{2(1-c)+2(1-t) d+3 t x+(2-t) z\}^{2}\right]
\end{aligned}
$$

Lemma 3. 1) The functions $\Pi_{u p}^{n}$, $\Pi_{d n}^{n}$ are pseudoconcave for all $d$, $t$.

2) The functions $\Pi_{u p}, \Pi_{d n}$ are pseudoconcave for all $t$ if $d \geq c / 5$.

3) If $d<c / 5$, there exists a $t^{*} \in(1 / 2,1)$ such that the functions $\Pi_{u p}, \Pi_{d n}$ are pseudoconcave.

Proof. 1) The fact that $\Pi_{u p}^{n}$ is pseudoconcave can be proved by showing that the cross derivative, $\partial_{z x} \Pi_{u p}^{n}$, is decreasing in $t$ and positive at $t=1$. In fact one can check that

$$
\partial_{t}\left(\partial_{z x} \Pi_{u p}^{n}\right)=\frac{-2(1-c-z)-(8 t-1)(z-x)-6\{t d+(1-t) x\}}{6(c-d)}-\frac{(2 t-1)(x-d)^{3}}{(c-d)(z-d)^{2}} .
$$

Since $1-c>z$ and $z>x$ (upward deviation), one has $\partial_{t}\left(\partial_{z x} \Pi_{u p}^{n}\right)<0$. The value of $\partial_{z x} \Pi_{u p}^{n}$ at $t=1$ is

$$
\left.\partial_{z x} \Pi_{u p}^{n}\right|_{t=1}=\frac{(x-d)\left(2 d^{2}+6 d x-3 x^{2}-10 d z+5 z^{2}\right)}{12(c-d)(z-d)^{2}} .
$$

The second term in the numerator is an increasing function of $z$ and takes the value $2(x-d)^{2}$ when $z=x$. Hence $\partial_{z x} \Pi_{u p}^{n}$ at $t=1$ is positive for $z>x$.

The fact that $\Pi_{d n}^{n}$ is pseudoconcave can be proved by checking that the cross derivative, $\partial_{z x} \Pi_{d n}^{n}$, is positive:

$$
\partial_{z x} \Pi_{d n}^{n}=\frac{t\{2(1-c)+4 z+t(3 x-2 z-d)\}}{6(c-d)}>0 .
$$

2) The fact that $\Pi_{u p}$ is pseudoconcave for $d \geq c / 5$ can be proved by checking that (i) the cross derivative, $\partial_{z x} \Pi_{u p}$, is a concave quadratic function of $t$, that (ii) $\partial_{z x} \Pi_{u p}$ takes 
the same (positive) value as $\partial_{z x} \Pi_{u p}^{n}$ at $t=1$ and decreasing in $t$ at $t=1$, and that (iii) $\Pi_{u p}$ takes the value $(5 z-c) / 8(c-d)$ at $t=1 / 2$. The cross derivative is

$$
\begin{aligned}
\partial_{z x} \Pi_{u p}= & \frac{1}{12(c-d)(z-d)^{2}}\left[2\{3-(2-t) t\} d^{3}-3(2 t-1)^{2} x^{3}\right. \\
& -6 c(1-t)^{2}(z-d)^{2}+(2 t-1)(4 t+1) x z^{2}+2(8-t)(1-t) z^{3} \\
& +2 d^{2}\{5(1-(1-t) t) z-(5-(17-14 t) t) x\}+9(2 t-1)^{2} d x^{2} \\
& \left.-2(2 t-1)(4 t+1) d x z-\{29-8(4-t) t\} d z^{2}\right]
\end{aligned}
$$

The derivative of $\partial_{z x} \Pi_{u p}$ w.r.t. $t$ at $t=1$ is

$$
\left.\partial_{t}\left(\partial_{z x} \Pi_{u p}\right)\right|_{t=1}=\frac{-6 d-7(z-x)}{6(c-d)}-\frac{(x-d)^{3}}{(c-d)(z-d)^{2}}<0 .
$$

3) This follows from the proof of 2) and the continuity of $\partial_{z x} \Pi_{u p}$. In fact, if $\partial_{z x} \Pi_{u p}>0$ for some $t^{*}$, it is positive for all $t>t^{*}$.

Proposition 4. Adding the royalty scheme increases the innovator's expected profit, $G>G_{n}$, for all parameters permitted in Lemma 3.

Proof. We show that the difference of the innovator's expected profit, $\Delta G(t):=G-$ $G_{n}$, is a concave quadratic function of $t$, which takes positive values at $t=\frac{1}{2}$ and 1 . One can easily find that

$\Delta G(t)=-\frac{(c-d)^{2}}{9} t^{2}+\frac{c-d}{36}(12-15 c-13 d) t+\frac{1}{36}\left\{5 c^{2}-2 c(2+9 d)+(16-11 d)\right\}$.

Hence,

$$
\begin{aligned}
\Delta G(1) & =\frac{1}{18}\left[c(4-7 c)+2(1-2 c) d-d^{2}\right] \\
\Delta G\left(\frac{1}{2}\right) & =\frac{1}{72}\left[c(4-7 c)+10(2-3 c) d-11 d^{2}\right] .
\end{aligned}
$$

Both are concave functions of $d$ taking positive values at $d=0$ and $d=c$;

$$
\begin{gathered}
\left.\Delta G(1)\right|_{d=0}=\frac{c(4-7 c)}{18}>0,\left.\quad \Delta G\left(\frac{1}{2}\right)\right|_{d=0}=\frac{c(4-7 c)}{72}>0 \\
\left.\Delta G(1)\right|_{d=c}=\left.\Delta G\left(\frac{1}{2}\right)\right|_{d=c}=\frac{c(1-2 c)}{3}>0 .
\end{gathered}
$$

\section{References}

Bagnoli, M., Bergstrom, T., 2005. Log-concave probability and its applications. Economic Theory 26, 445-469.

Das Varma, G., 2003. Bidding for a process innovation under alternative modes of competition. International Journal of Industrial Organization 21, 15-37. 
Fan, C., Jun, B., Wolfstetter, E., 2009a. Auctioning a process innovation when losers' bids determine royalty rates. Working paper, Department of Economics, Humboldt University at Berlin.

Fan, C., Jun, B., Wolfstetter, E., 2009b. Auctioning a process innovation when losers' bids determine royalty rates. Working paper, Department of Economics, Humboldt University at Berlin.

Giebe, T., Wolfstetter, E., 2008. License auctions with royalty contracts for (winners and) losers. Games and Economic Behavior 63, 91-106.

Goeree, J., 2003. Bidding for the future: Signaling in auctions with an aftermarket. Journal of Economic Theory 108, 345-364.

Goeree, J., Offerman, T., 2003. Competitive bidding in auctions with private and common values. Economic Journal 113, 598-613.

Harstad, R. M., Levin, D., 1985. A class of dominance solvable common-value auctions. Review of Economic Studies 52, 525-528.

Jehiel, P., Moldovanu, B., 2000. Auctions with downstream interaction among buyers. RAND Journal of Economics 31, 768-791.

Kamien, M., Tauman, Y., 1984. The private value of a patent: a game theoretic analysis. Journal of Economics 4, 93-118.

Kamien, M. I., 1992. Patent licensing. In: Aumann, R., Hart, S. (Eds.), Handbook of Game Theory. Vol. I. Elsevier Science, pp. 331-354.

Kamien, M. I., Tauman, Y., 1986. Fee versus royalties and the private value of a patent. Quarterly Journal of Economics 101, 471-491.

Katz, M. L., Shapiro, C., 1985. On the licensing of innovations. RAND Journal of Economics 16 (4), 504-520.

Katz, M. L., Shapiro, C., 1986. How to license intangible property. Quarterly Journal of Economics 101, 567-589.

Klemperer, P., 1998. Auctions with almost common values: the "wallet game" and its applications. European Economic Review 42, 757-769.

Sen, D., 2005. Fee versus royalty reconsidered. Games and Economic Behaviour 53, 141-147.

Sen, D., Tauman, Y., 2007. General licensing schemes for a cost-reducing innovation. Games and Economic Behavior 59, 163-186.

Wilson, R. B., 1977. A bidding model of perfect competition. Review of Economic Studies 44, 511-518. 